\section{Health for all in the twenty-first century ${ }^{1}$}

${ }^{1}$ This paper is condensed from WHO Document A51/5.
Health for All (HFA) was defined in 1977, when the Thirtieth World Health Assembly decided that governments and $\mathrm{WHO}$ should focus primarily on attaining by the year 2000 a level of health that permits every individual to lead a socially and economically productive life. The Declaration of Alma-Ata, adopted in 1978, stated that primary health care (PHC) is the key to attaining HFA. The call for HFA is fundamentally a call for social justice.

HFA can be interpreted differently according to each country's social, economic, and health conditions. However, all people in all countries should have a level of health that will permit them to work productively and participate actively in the social life of their community.

HFA in the twenty-first century builds on past achievements, guides action and policy for health at international, regional, national, and local levels, and identifies global priorities and targets for the first two decades of the twenty-first century. Most of all, it takes into account the dramatic global changes of the past 20 years.

\section{Old and new challenges}

Over the past two decades, governments and nongovernmental organizations have increasingly accepted HFA in their efforts to improve health. Access to basic PHC has steadily increased. These policies and actions have contributed to the worldwide decline in infant and child morbidity and mortality and to substantial increases in life expectancy at birth.

However, a number of factors have hampered progress, among them:

- insufficient political commitment to HFA;

- failure to achieve equity in access to all PHC elements;

- the continuing low status of women;

- slow socioeconomic development;

- difficulty in achieving intersectoral action for health;

- unbalanced distribution of, and weak support for, human resources;

- widespread inadequacy of health promotion activities; 


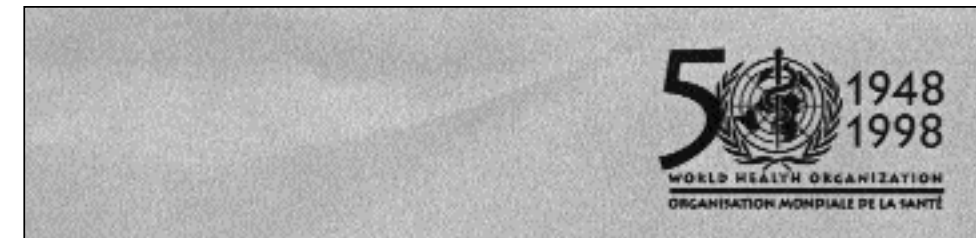

\section{WORLD HEALTH DECLARATION}

We, the Member States of the World Health Organization (WHO), reaffirm our commitment to the principle enunciated in its Constitution that the enjoyment of the highest attainable standard of health is one of the fundamental rights of every human being, in doing so, we allirm the dignity and worth of every person, and the equal rights, equal duties and shared responsibilities of all for health.

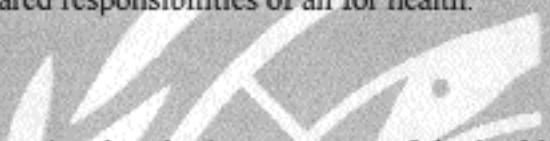

II

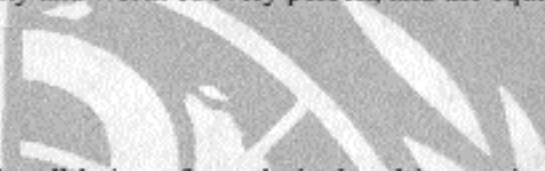

We recognize that the improvement of the health and well-being of people is the ultimate aim of social and economic development. We are committed to the ethical concepts of equity, solidarity and social justice and to the incorporation of a gender perspective into our strategies. We emphasize the importance of reducing social and economic inequities in improving the health of the whole population. Therefore, it is imperative to pay the greatest attention to those most in need, burdened by ill-health, receiving inadequate services for health or affected by poverty. We reaffirm our will to promote health by addressing the basic determinants and prerequisites for health. We acknowledge that changes in the world health situation require that we give effect to the "Health-for-All Policy for the twenty-first century" through relevant regional and national policies and strategies.

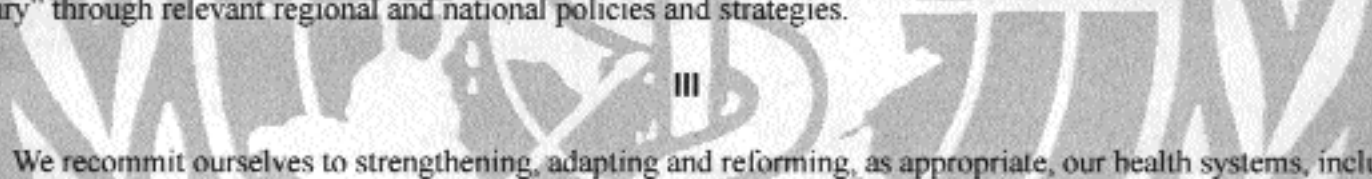

We recommit ourselves to strengthening, adapting and reforming, as appropriale, our bealth systems, including essential public health functions and services, in order to ensure universal aceess to health services that are based on scientific evidence, of good quality and within affordable limits, and that are sustainable for the future. We intend to ensure the availability of the essentials of primary health care as defined in the Declaration of Alma-Ata' and developed in the new policy. We will continue to develop health systems to respond to the current and anticipated health conditions, socioeconomic circumstances and needs of the people, communities and countries concerned, through appropriately managed public and private actions and investments for health.

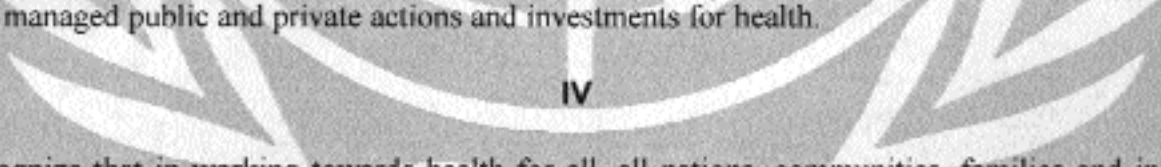

We recognize that in working towards health for all, all nations, communities, families and individuals are interdependent. As a community of nations, we will act together to meet common threats to health and to promote universal well-being.

We, the Member States of the World Health Organization, hereby resolve to promote and support the rights and principles, action and responsibilities enunciated in this Declaration through concerted action, full participation and partnership, calling on all peoples and institutions to share the vision of health for all in the twenty-first century, and to endeavour in common to realize it.

${ }^{1}$ Adopted at the International Conferenee on Primary Health Care, Alma-Ata, 6-12 September 1978, and endorsed by the Thirty-second World Ilealth Assembly in resolution WHA32.30 (May 1979). 
- weak health information systems and absence of baseline data;

- pollution, poor food safety, and lack of safe water supply and sanitation;

- rapid demographic and epidemiological changes;

- inappropriate use of, and allocation of resources for, high-cost technology; and

- natural and man-made disasters.

Poverty and growing inequities. Despite significant health gains over the past 50 years, certain gaps between and within countries have widened. The debt crisis of the 1980s made many countries reduce their support for health and social services. Health has suffered most where economies have been unable to secure adequate income for all, where social systems have collapsed, and where natural resources have been poorly managed.

The number of people living in absolute poverty is growing and is now nearly 1300 million. Poverty is a major cause of undernutrition and illhealth; it undermines the effectiveness of health services. The poor often live in unsafe and overcrowded housing, underserved rural areas, or periurban slums. They are more likely than the well off to be exposed to pollution and other health risks at home, at work, and in their communities. They are also more likely to consume insufficient food and food of poor quality, to smoke tobacco, and to be exposed to other risks harmful to health. The increasing gaps between rich and poor threaten social cohesion and, in many countries, contribute to violence and psychosocial stress.

Demographic and epidemiological changes. Increased life expectancy, lower birth rates, and a rise in noncommunicable diseases, combined with exposure to new threats, define the challenges for the future. Sheer population numbers in some countries and high resource consumption in others compromise the chances of meeting the future needs of the world's people.

All populations are aging, a process often leading to an increase in noncommunicable diseases, disability, and mental disorders. This trend is already exerting pressure on social support systems and requiring a shift in health services. Also, in some countries the demographic transition will result in an absolute increase in the number of young people and, consequently, in greater pressures on the health system, educational services, and employment.
Urbanization has improved the quality of life and health in many countries. However, uncontrolled urban growth fosters the spread of infectious diseases. Overcrowding and poor working conditions can lead to anxiety, depression, and chronic stress. Changes in family structure and living arrangements have compromised many people's health and ability to cope with health and social problems. Disruption of traditional rural cultures has eroded many social support systems.

Communicable diseases, malnutrition, and maternal mortality. Despite a worldwide decline in communicable diseases, new and old infectious diseases still threaten global health. Travel and trade, urbanization, migration, and microbial evolution may amplify these diseases. The burden of infectious disease remains particularly high among children in the developing world, due to the interaction of perinatal factors, poor nutrition, and diseases such as acute respiratory infections, diarrhea, measles, and malaria.

Efforts to reduce malnutrition in children in the poorest countries have stagnated. Almost a billion people cannot meet their basic daily requirements for energy and protein, and more than two billion suffer from micronutrient deficiencies. Maternal deaths are still unacceptably common in developing countries.

Noncommunicable diseases. Noncommunicable diseases include major causes of death such as ischaemic heart disease, diabetes, and cancer, as well as disability, such as that produced by mental disorders. Today, they contribute significantly to the global burden of disease. If current trends in tobacco use, a high-fat diet and obesity, and other health risks continue, such diseases will become the dominant causes of death, disease, and disability worldwide by the 2020s. Tobacco use is a risk factor for some 25 diseases whose impact is still not fully appreciated.

Violence, injuries, and social disintegration. Violence takes several forms and includes tribal or ethnic conflict, gang warfare, and family violence. In some countries, exposure to violence in the entertainment media, combined with easy access to weapons and use of alcohol and illicit drugs, has contributed to an increase in violence. In many 
societies, there is concern about social disintegration stemming from the weakening of human relationships based on sharing and caring, of the bonds sustaining and nurturing intergenerational relationships, and of the family as a social unit. Unemployment, alcohol dependence, and mental disorders are on the rise. Injuries are likely to rise with increased use of motor vehicles, urbanization, and industrialization.

\section{New trends that will influence health}

Globalization. The dramatic growth in trade, travel, and migration, together with developments in technology, communications, and marketing, has resulted in substantial gains for some groups and severe marginalization for others. The spread of information technologies and advances in biotechnology will increasingly help in detecting, preventing, and mitigating the impact of disease outbreaks, famine, and environmental health threats, and in bringing health services and education to many more people. However, there is concern that increased trade in products harmful to health threatens the health of populations, particularly in low-income countries. Increased transnational trade in food and the mass movement of people constitute additional global threats to health. At the same time, there is concern that globalization threatens the survival of cultural and ethnic diversity and reduces public investment for health.

Environmental and industrial changes. Global environmental hazards, such as air pollution, ozone depletion, climate change, loss of biodiversity, and the cross-border movement of hazardous products and wastes, have adverse impacts on health. Unplanned and poorly controlled industrialization, combined with inefficient use of energy in transport, manufacturing, and construction, poses threats to air quality in most rapidly growing cities. Indoor air pollution is a major cause of morbidity and premature death. Improper food processing is associated with foodborne disease, diarrheal diseases, and other conditions. Hazardous occupations, unsafe working practices and conditions, and increased competitiveness in changing economies contribute to stress and other health problems.

The changing role of the State. Relationships between countries are influenced by a host of factors, particularly the spread of market forces and the increasing interdependence of countries. Governments must function under pressures from many sources to bring national policies in line with global and regional agreements. Corruption has eroded public confidence in many governments and, in some countries, even the structure of government has collapsed.

\section{HEALTH FOR ALL IN THE TWENTY-FIRST CENTURY}

\section{Values, goals, and targets of Health for All in the twenty-first century}

Values. The HFA vision is based on the following key values:

Ethics: Respect for individual choice, personal autonomy, and the avoidance of harm applies to both individual and social aspects of health care and research. Advances in science and technology, medicine, engineering, and communications offer untold opportunities to influence health, but firm ethical principles are needed to guide scientific and technological developments and their application.

Equity: An equitable health system ensures universal access to adequate quality care. At every level, the measurement of inequities is the startingpoint for policy development and action. Equity and solidarity should form the basis for international technical cooperation, favoring populations and countries with the greatest burden of poverty and ill health.

A gender perspective: A gender perspective acknowledges the effects of the socially, culturally, and behaviorally determined relationships, roles, and responsibilities of men and women. Specific aspects include:

- performing gender analyses and encouraging gender awareness;

- attending to the special needs of girls and boys, women and men, throughout the life span;

- supporting the human rights, dignity, self-worth and abilities of girls and women;

- creating opportunities for full participation of women with men in decision-making at all levels.

Goals and targets. The goals of HFA are:

- an increase in life expectancy and quality of life for all; 
- improved equity in health between and within countries;

- universal access to sustainable health systems and services.

An initial set of targets will guide the implementation of the HFA policy for the first two decades of the next century. Regional and national targets will be developed within the framework of the global policy. They should be measurable, timebound, and feasible. All targets should be reviewed periodically.

The strengthening of health information systems since 1981 means that the targets for 2020 are more firmly based on evidence than previous targets. They are:

By 2005:

- Health equity indices will be used within and between countries as a basis for promoting and monitoring equity in health. Initially, equity will be assessed based on child development.

- All Member States will have operational mechanisms for developing, implementing, and monitoring policies that are consistent with this HFA policy.

By 2010:

- Leprosy will be eliminated and transmission of Chagas' disease interrupted.

- All people will have lifelong access to comprehensive, essential, quality health care, supported by essential public health functions.

- Appropriate global and national health information and surveillance systems will be established.

- Research policies and institutional mechanisms will be operational at global, regional, and country levels.

By 2020:

- Maternal mortality in all countries will be $<100$ per 100000 live births; under-five or child mortality rates will be $<45$ per 1000 live births; and life expectancy will exceed 70 years.

- The worldwide burden of disease will be substantially decreased. This will be achieved by implementing sound disease-control programs aimed at reversing the current trends of increasing incidence and disability caused by tuberculosis, HIV / AIDS, malaria, tobacco-related diseases, and violence/trauma.
- The following diseases and conditions will be eliminated: measles, lymphatic filariasis, trachoma, and vitamin A and iodine deficiencies.

- All countries will have made major progress in providing safe drinking-water, adequate sanitation, food, and shelter.

- All countries will have introduced strategies that strengthen health-enhancing lifestyles and weaken health-damaging ones, through regulatory, economic, educational, organizational, and community-based programs.

\section{Policy basis for action}

The goals of HFA will be realized through the implementation of two policy objectives: making health central to human development and developing sustainable health systems to meet the needs of people.

Making health central to human development. The purpose of human development is to permit people to lead economically productive and socially satisfying lives. This approach places health firmly at the center of the development agenda.

Developing sustainable health systems to meet the needs of people. Health systems must be able to respond to the health and social needs of people over their life span. Efforts should be directed towards clearly identifying health needs and organizing comprehensive services within a welldefined population base.

Actions for good health start in the home. Health services should complement the actions of individuals and families by promoting healthy living and providing information and access to quality health care, and by supporting public health functions.

Building on primary health care, health systems should be: community-based and comprehensive, with preventive, promotive, curative, and rehabilitative components; available continuously; adequately financed; closely linked at all levels to social and environmental services; and integrated into a wider referral system.

A sustainable health system will actively encourage community participation in policy development. A socially sensitive health system will recognize the economic, sociocultural, and spiritual values of different groups, the variety of systems 
of health and healing, and their potential for coexistence and mutual enrichment. Besides extending life and improving health, health systems must relieve pain and suffering, provide compassionate care to those with incurable disease, and try to ensure a peaceful and dignified death.

The social aspect of creating health care systems involves integrating health into daily community life, developing community support, and maximizing people's participation in maintaining the health of their families and communities. Governments must ensure that health systems are financially sustainable and accountable, with universal access and quality.

The role of the World Health Organization. As the world's health advocate, WHO will provide global leadership for the attainment of HFA. WHO will promote international collective action for health by developing global ethical and scientific norms and standards; develop international instruments that promote and protect global health; facilitate technical cooperation among countries; strengthen decision-making through appropriate health information systems; establish active surveillance systems; strengthen global research capacity; provide leadership for the eradication, elimination, and control of selected diseases; and provide technical support to prevent public health emergencies and assist with postemergency rehabilitation.

\section{FULFILLING THE VISION: ACTIONS FOR IMPLEMENTATION OF THE POLICY}

\section{Actions needed to make health central to development}

Four lines of action are required to address the determinants of health and to make health central to human development.

Combat poverty. Accelerated human development and economic growth in both the public and private sectors are needed to lift the poorest people and communities out of poverty. Such growth must be backed by substantive and sustained international support for the poorest countries, including integrated development plans with debt reduction and provision of credit. Everywhere, ethical economic policies that enhance equity are essential for sustainable economic growth and human development.
Health interventions must be linked to the improved education of girls and the provision of a basic public health infrastructure and essential health services. Ready access by the poor to quality health care services, through outreach to their homes if required, should be supported as an essential component of poverty-reduction programs.

Collaboration between health systems and agricultural, trade, financial, food and nutrition, education, and industry sectors is essential for sustained alleviation of poverty. Disease-control programs may have a considerable impact where one or a few diseases make a major contribution to poverty. Examples are the control of onchocerciasis in West Africa and of malaria in many areas, which have contributed significantly to food production and income generation. Combined food aid and deworming programs can raise children's scholastic performance and school attendance.

Promote health in all settings. Individuals, families, and communities need knowledge and skills to keep themselves healthy. Health promotion must adjust to the social, cultural, political, legal, and spiritual environments in which people live, work, play, and learn. Social action can help to protect the young from violence and substance abuse, ensure that working conditions are conducive to health, promote healthy diets and recreation, and create a school environment that is supportive of learning, good health, and personal growth. The media can play a greater role in disseminating health information and advocating health and health practices among even the most remote families.

Align sectoral policies for health. The policies of all governmental sectors that affect health directly or indirectly-including, among others, agriculture, housing, energy, water, transport, education, and justice-need to be aligned to maximize opportunities for health promotion and protection. Health professionals should be willing to negotiate with these other sectors for mutually beneficial policies. Multidisciplinary research is required to identify opportunities for health promotion and protection through intersectoral action.

Stronger joint action by health systems and the education sector could enhance the overall health status of populations and reduce health and economic inequalities. Fiscal policies that contribute to health-for instance, discouraging the use of harmful products and stimulating adoption of healthy lifestyles-should be furthered. They can 
help to retard and even reverse increases in noncommunicable diseases and trauma.

Agricultural policies can incorporate specific disease prevention measures in irrigation schemes, promote integrated pest management to minimize the use of toxic chemicals, establish land-use patterns that facilitate human settlements in rural areas, encourage substitution for crops that harm health, and ensure the production of safe and sufficient foods. Energy policies should support the use of cleaner energy sources and more efficient energy use. National policies to address population growth will integrate strategies to improve the status of women, particularly through access to education and primary and reproductive health care programs and equal participation in decision-making.

Include health in planning for sustainable development. Health considerations must receive the highest priority in sustainable development plans. Promotion and protection of human health and well-being should be a primary reason for all aspects of development.

Non-renewable resources have been dangerously over-exploited, while renewable resources are being consumed on a non-sustainable basis. Conventions and actions that discourage or prevent severe environmental degradation will benefit the health of future generations.

The introduction of health indicators into environmental impact assessment will improve decision-making in the health and environment sectors. The health consequences of environmental changes must be quantified and used to assess progress towards sustainable development.

\section{Essential functions of sustainable health systems}

A life-span approach to health emphasizes interventions with a preventive and caring potential that can extend from birth to death. Many conditions and behaviors can be prevented by investment in early childhood development, leading to important improvements in health later in life.

Life-span care should be available in local communities, within a health system that emphasizes quality of prevention, diagnosis, treatment, and rehabilitation. Local and district health services must be able to provide essential drugs and other services to meet community needs. They should be linked electronically, and by permanently available transport, to referral centers.
Preventing and controlling disease, and protecting health. Community-based population-oriented disease prevention and control and health protection services benefit everyone. Priority should be given to endemic and commonly occurring infections, noncommunicable diseases, injuries, and violence. Services should be decentralized as much as possible.

Disease prevention and health protection services in the workplace are essential to improving the health of workers. The current emphasis on preventing exposure to specific agents and on promoting safety at work should be extended to cover all preventable conditions in the workplace. Where people work at home, their occupational health needs should be met by local or district health services.

Promoting legislation and regulations in support of sustainable health systems. National laws should set the basis for collective action for health, protect vulnerable and disadvantaged people from adverse economic effects, and define the boundaries between government and its partners. Health ministries and departments are responsible for developing policies and priorities that reflect people's needs: by setting standards and norms, ensuring that supportive legislation and regulations are adopted, and informing the public about its rights and responsibilities.

Legislation that promotes health includes measures relating to: environmental standards, food safety, bans on tobacco advertising and sponsorship, restrictions on alcohol promotion, bans on access to certain weapons, measures for consumer protection, and the entitlements of people to health care. Legislation is also needed to: help control violence and injury; prohibit the creation of environmental hazards; ensure that ethical practices are followed in medical care and research; provide a regulatory framework for private-sector health care and intersectoral action for health; ensure the safety of pharmaceuticals and foods; and protect consumers and health care providers.

Developing health information systems and ensuring active surveillance. A hallmark of a sustainable health system is its emphasis on active surveillance and monitoring. Global, regional, national, and local surveillance, and monitoring and early warning systems will alert the public to impending threats to health. Every integrated system of active surveillance and monitoring for health should cover the following areas: infectious diseases; health status and trends, including birth and death rates; implementation of international norms, standards and regulations; 
progress in reducing health inequities; performance of essential public health functions; the impact of various lifestyles on health status; the health impact of human rights abuse; transnational health problems; and sectoral impacts on health.

Fostering the use of science and technology. Advances in science and technology are likely to provide unprecedented benefits for all in the twentyfirst century. Rapid progress in several fields over the coming decades should allow poorer countries to take advantage of developments in technology and benefit from the experiences of other countries.

Closer partnerships between science and technology research and development, between users and innovators, and between the private and public sectors will increase the application of scientific innovation to improved health worldwide. Researchers and research funding agencies must be encouraged to share expertise and resources in a spirit of international solidarity.

In assessing and promoting new technologies for health, the following should be considered: the ability to contribute to quality of life and health; the potential to promote equity; the respect for privacy and individual autonomy; and the degree of focus on determinants of health. An effort must be made to adopt a long-term time-frame and broad view with respect to technology transfer, as the benefits and applications of technology are not always immediately understood, realized, or affordable.

Building and maintaining human resources for health. Support by the State, WHO, and their partners in health training institutions should reflect the need for ongoing and comprehensive capacity-building for health. A culture of health that respects and supports the right to health, ethics, equity, and gender equality is fundamental. Professional codes of conduct should be consistent with HFA values.

Human resource planning should recognize the need to consider changing mixes of health care providers working in a multidisciplinary, collaborative fashion. Technical cooperation and national and international training opportunities have to be strengthened in order to fill gaps in the supply of public health professionals. To serve the need of the public for better information about all aspects of health, greater attention should be given to training in communications, health promotion skills, care giving, and community assessment. Telecommunications linkages offer new opportunities for distance-learning and diagnostic support. They can promote accelerated development of human resources in poor countries and communities.

The health sector should develop national policies that contribute to self-sufficiency in human resource development, and appropriate career development and deployment of the health workforce. Global and regional policies must address broader human resource issues, such as the transnational movement of health professionals, the provision of training, the need for international harmonization of education and service standards, and the use of appropriate regulatory and financial mechanisms to maintain and strengthen national capacity.

Securing adequate and sustainable financing. Government action and regulations are needed to secure an adequate level of financing, promote cost containment and fiscal discipline, provide lists of essential drugs and technology, and ensure that national resources are used equitably. When the government has the major mandate for health systems, or is their main provider of funds, there is more likely to be equity of access, cost containment, and a strong emphasis on preventive and promotive services.

In many of the poorest countries, financing from community sources and international donors is required to support essential health system functions, particularly those that benefit poor people. In middle-income countries, deriving a large share of financing from a pre-paid source of revenue improves the chances for achieving equitable and efficient health services. In high-income countries, increased health care costs may not yield health gains. In all countries, containment measures should be considered in order to maximize cost-effectiveness.

The costs of ensuring access to essential health care for everyone, as well as the effects of rationing, should be distributed fairly across the population, according to need. However, shifting health care costs from the public sector to individuals and families should be done with caution.

\section{Keys to successful implementation of Health for All in the twenty-first century}

Strengthening capacity for policy-making. Translation of the HFA policies into action must be considered in the context of the overall economic and social situation of a country or locality. Each country will select the best mix of policies to achieve HFA, consistent with its national needs, capacities, and priorities.

Governments need a strong policy-making capacity to address the major challenges con- 
fronting them. Above all, they must develop a supportive organizational culture that encourages health workers to innovate and move steadily towards clearly defined policy goals and targets. Decisions should be assessed for their short-, medium-, and long-term implications, with the ultimate goal of achieving sustainable outcomes. For policy to be based on scientific evidence, a solid research base in health and epidemiology is needed, together with related information on public preferences as well as on the availability of resources.

Good governance. Governance is society's system for organizing and managing the affairs of its diverse sectors and partners in order to achieve its goals. Hallmarks of good governance for health are transparency, accountability, and incentives that promote participation. Criteria used for decisionmaking, from priority-setting to allocation of resources, are made public and the results of monitoring and evaluation of implementation are widely distributed.

National governments have an obligation to ensure that health is explicitly considered in the development of public policy. Decentralized decision-making for health will help to ensure that local needs are considered. Local participatory planning, full use of local capacity and resources, and more effective collaboration in bringing environmental, social and economic services closer to people will strengthen community ownership of those services and increase their utilization.

International and foreign policy must be broader-based, with greater emphasis on international health security and its contribution to sustainable peace. Foreign policies should include public health approaches to disease prevention and health promotion.

Regional economic, political, and development alliances, as well as new bilateral and multilateral bodies, should be formed with a view to creating new opportunities for regional governance for health. It will be important to ensure that policies and actions are targeted at the level at which they can be of greatest benefit to health.

Setting priorities for action. A wide range of strategies are available to improve health, but resources are limited. Governments must select priorities. Five possible levels of financial decisionmaking for health systems are:

- macro-level of funding for health systems and services;
- distribution of the budget among different geographical areas and services;

- allocation of resources to particular forms of treatment;

- choices concerning which patients should receive treatment;

- decisions on how much to spend on individual patients.

Priorities for action in a given population will be determined by the relative importance of the different health problems, in terms of: epidemiological measures of the burden of disease or suffering, the effectiveness (and cost-effectiveness) of interventions to improve health and reduce inequity, the likely trends in the absence of action, the capacity of the health sector to act or promote intersectoral action, and public support.

Priorities should be set using an open, consultative approach involving the public and key partners for health. Regular dialogue and the exchange of views between the groups will lead to the development of a shared understanding of the major problems and options for action. The views of the poor, women, marginalized groups, patients, and disabled people are critical.

Partnerships for health. Partnerships are needed between the multiple levels and sectors concerned with health and will be a primary component of HFA implementation. Productive partnerships will enable different ideologies, cultures, and talents to come together in a way that creates energy and stimulates the imagination in working towards improved health.

Community partnerships and the development of skills, with the aim of increasing both the options available to individuals and countries and the control they exercise over those options, constitute the essence of HFA. People's direct and indirect participation in the promotion and maintenance of their health and that of their families and communities lies at the core of people-centered approaches to development.

Both formal partnerships for health and community-based informal networks at different levels are needed. WHO and governments should consider developing guidelines with the private sector aimed at ensuring that new partnerships are mutually beneficial and always health-promoting. Partnerships can draw upon the energy and vitality of civil society, particularly nongovernmental organizations, to develop environments that support health. 
Global action in support of national health. Global action and cooperation between countries should aim at securing the benefits of globalization for the health of all on an equitable basis and at preventing or minimizing health threats. Global public health action includes active surveillance, support for research on poverty and health, and development of global ethical and scientific norms and standards. It includes prevention, control, eradication, or elimination of diseases and their risk factors that constitute transnational threats to health and are amenable to interventions. In addition, liberalization of trade calls for greater compatibility in policy objectives to be developed between international and intergovernmental agencies and multinationals involved in trade and health.

Evaluation and monitoring. Evaluation, which is the measurement of performance against objectives, should play a key role in strengthening the policy process and serve as the ultimate test of success. The process of evaluation should be integrated with goal-setting and the development of targets and indicators.

\section{SINOPSIS}

\section{Salud para todos en el siglo XXI}

Salud para todos en el siglo XXI renueva el compromiso con el concepto y la visión de salud para todos, tal como se definió en 1977, cuando la 30a Asamblea Mundial de la Salud resolvió que el principal objetivo social de los gobiernos y de la OMS en los decenios venideros debería ser "alcanzar para todos los ciudadanos del mundo en el año 2000 un grado de salud que les permita llevar una vida social y económicamente productiva". Este proceso, que se interpreta de forma diferente según las características sociales, económicas y de salud de cada país, ha contribuido a la mejora paulatina del acceso a la atención primaria de salud, a la disminución mundial de la morbilidad y la mortalidad infantiles y a un aumento considerable de la esperanza de vida. Aun así, todavía hay muchos problemas que resolver. Desde 1990, el sistema de las Naciones Unidas ha convocado nueve conferencias mundiales en las que se han acordado por consenso universal las prioridades de un programa de trabajo para el futuro, incluido el apoyo pleno del logro de salud para todos. Los nuevos enfoques giran en torno al desarrollo centrado en el ser humano, lo cual comprende erradicar la pobreza, satisfacer las necesidades básicas y proteger los derechos humanos, incluido el de la salud. 